\title{
An SV-GMR Needle Sensor-Based Estimation of Volume Density of Magnetic Fluid inside Human Body
}

\author{
C. P. Gooneratne, ${ }^{1}$ S. C. Mukhopadhyay, ${ }^{2}$ and S. Yamada ${ }^{1}$ \\ ${ }^{1}$ Institute of Nature and Environmental Technology (K-INET), Kanazawa University, Kakuma, Kanazawa 920-1192, Japan \\ ${ }^{2}$ Division of Biological Measurement and Applications, Institute of Information Sciences and Technology, Massey University, \\ AG-HORT Building AH 3.74, Palmerston North, New Zealand
}

Correspondence should be addressed to S. C. Mukhopadhyay, s.c.mukhopadhyay@massey.ac.nz

Received 18 June 2007; Accepted 25 July 2007

Recommended by Ignacio R. Matias

\begin{abstract}
A spin-valve giant magnetoresistive (SV-GMR) sensor of needle-type configuration is reported to estimate the volume density of magnetic fluid inside human body. The magnetic fluid is usually injected into human body to kill cancerous cell using hyperthermia-based treatment. To control the heat treatment, a good knowledge of temperature is very much essential. The SVGMR-based needle-type sensor is used to measure the magnetic flux density of the magnetic fluid inside the human body from which the temperature is estimated. The needle-type sensor provides a semi-invasive approach of temperature determination.
\end{abstract}

Copyright (c) 2008 C. P. Gooneratne et al. This is an open access article distributed under the Creative Commons Attribution License, which permits unrestricted use, distribution, and reproduction in any medium, provided the original work is properly cited.

\section{INTRODUCTION}

In recent times, due to the development of several novel sensors and sensing technologies, there is a tremendous progress in biomedical sensing. Modern biomedical sensors developed with advanced microfabrication and signal processing techniques are becoming inexpensive, accurate, and reliable [1]. To develop sensors to analyze cells at a single-cell level, it is very demanding to have high-performance sensing and instrumentation techniques $[2,3]$. A hyperthermia cancer treatment based on induction heating is achieved by injecting magnetic fluid with magnetic nanoparticles into the tumor. The potential benefit of using magnetic nanoparticles is the use of localized magnetic field gradients to attract the particles to a chosen site, to hold them there until the therapy is complete, and then to remove them [4]. To destroy the tumor, a time-varying magnetic field is applied to the nanoparticles for heating up the tumor. The applied heat depends on the amount of nanoparticles, magnetic flux density, exciting supply frequency, and the distance from the exciting coil to the location of tumor inside the body. To confirm that the tumor can be destroyed when exposed to heating, it is required to measure the temperature of the magnetic liquid inside the body. The control of temperature is a paramount important task in achieving success using this treatment method. But it is difficult to fabricate temperature sensor of very small size with sufficient accuracy to measure the temperature. So an indirect approach has been taken to measure the temperature in the tumor area inside the human body. This approach is based on the measurement of magnetic flux density of the magnetic liquid which is injected for treatment. In order to calculate temperature in an indirect way, knowledge about the density of nanoparticles is important before and during the treatment. This paper reports the development of a needle-type nanosensor based on the spinvalve giant magneto-resistive (SV-GMR) technique to measure the magnetic flux density inside the body via pricking the needle inside the body. The modeling and experimental results of flux-density measurement have been reported here. From the information of flux density, the temperature rise can be estimated to permit the delivery of controlled heating to precisely defined locations in controlled hyperthermia cancer treatment. The sensor needs to be used in a uniform magnetic field region which is provided by Helmholtz coils.

The complete paper is divided into six sections. After the introduction that covers the first section, sensor has been discussed in Section 2. The hyperthermia treatment and use of the sensor have been described in Section 3. The Helmholtz 


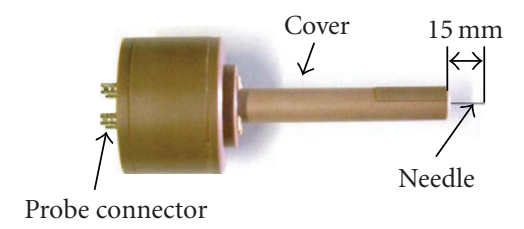

(a)

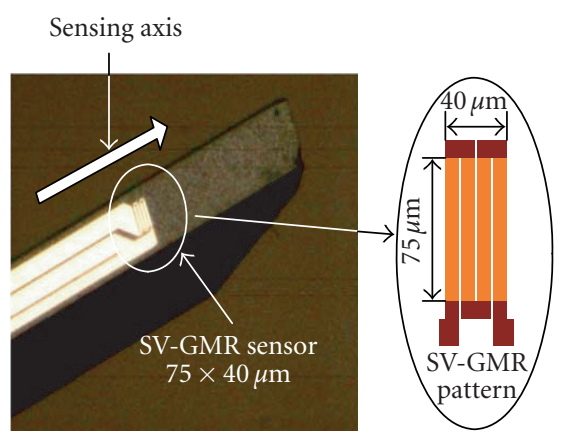

(b)

FiguRE 1: SV-GMR needle sensor.

coil, analytical results, and experimental results are provided in Sections 4 and 5. The experimental results are reported in Section 6. Finally the conclusion is summarized in Section 7.

\section{SV-GMR NEEDLE-TYPE SENSOR}

The SV-GMR sensor as shown in Figure 1 is used to estimate volume as well as weight density of the magnetic fluid inside human body. The needle-type construction paves for easier application for measurement inside the human body. The SV-GMR element with sensing area of $75 \mu \mathrm{m} \times 40 \mu \mathrm{m}$ is on the tip of the needle. Sensing direction is parallel to the needle. The volume/weight density of the magnetic fluid can be estimated by measuring and comparing the applied magnetic flux density (outside body) and the flux density in the magnetic fluid. A constant current of $0.5 \mathrm{~mA}$ is applied to the SV-GMR sensor. Figure 2 shows the AC characteristics of the sensor and the sensitivity is approximately $10 \mu \mathrm{V} / \mu \mathrm{T}$.

\section{SV-GMR SENSOR AND HYPERTHERMIA TREATMENT}

The block diagram representation of hyperthermia-based cancer treatment is shown in Figure 3. Hyperthermia treatment is a rapidly evolving cancer cure that has many advantages compared to other common methods of treatment. These include no side effects or pain for the patient, which is a major problem in treatments such as chemotherapy. A low/minimally invasive treatment method such as the one reported here takes less time for treatment compared to chemotherapy and radiotherapy. Even though the cancer cells do not die completely, they could quite possibly be more susceptible to treatments such as ionizing radiation or chemotherapy, allowing such therapy to be given in small doses.

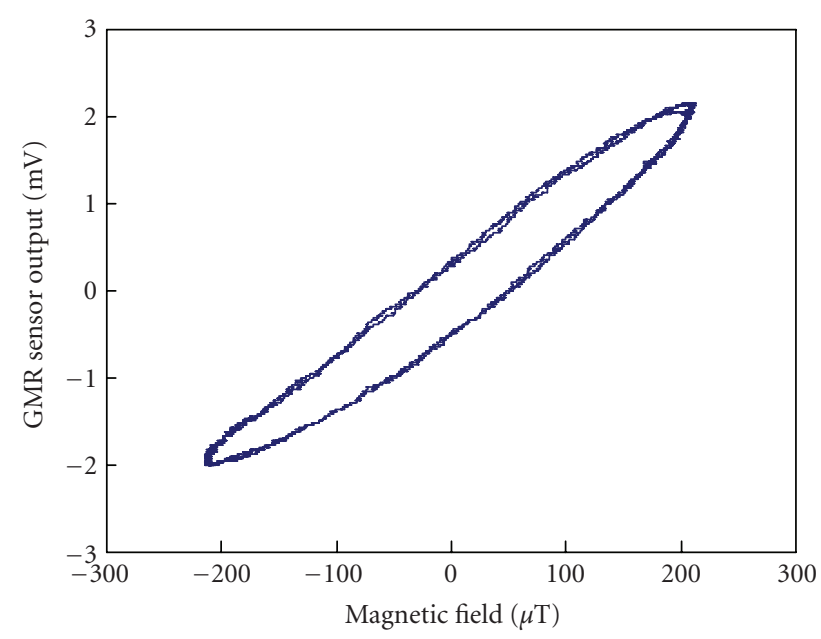

Figure 2: AC characteristics of the SV-GMR sensor.

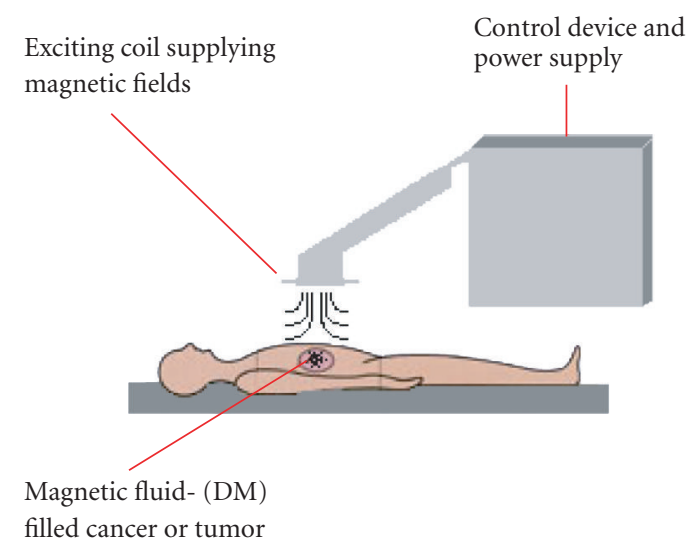

Figure 3: Hyperthermia treatment as a cancer therapy.

Hyperthermia is based on the principle that cancer cells are more sensitive to heat than normal healthy cells. Magnetic fluid with magnetic nanoparticles as shown in Figure 4 is inserted into the affected area.

When a time-varying external magnetic field is applied on the magnetic particle, magnetic particles absorb and generate heat due to hysteresis loss, and temperature is thus increased. It is essential to correctly control the temperature in hyperthermia treatment. The magnetic fluid density in the body is an important parameter to estimate the heat that needs to be given. This serves as a critical aspect in the success of the treatment. However when injected into the affected area, the magnetic fluid spreads inside the cancer cells and as a result, the volume as well as weight density of magnetic fluid decreases. As a consequence, it is vital to estimate the density of magnetic fluid in the body. The magnetic fluid weight density is the ratio of weight of magnetic nanoparticles in $1 \mathrm{~mL}$ volume to weight of magnetic nanoparticles and water in $1 \mathrm{~mL}$ volume. 


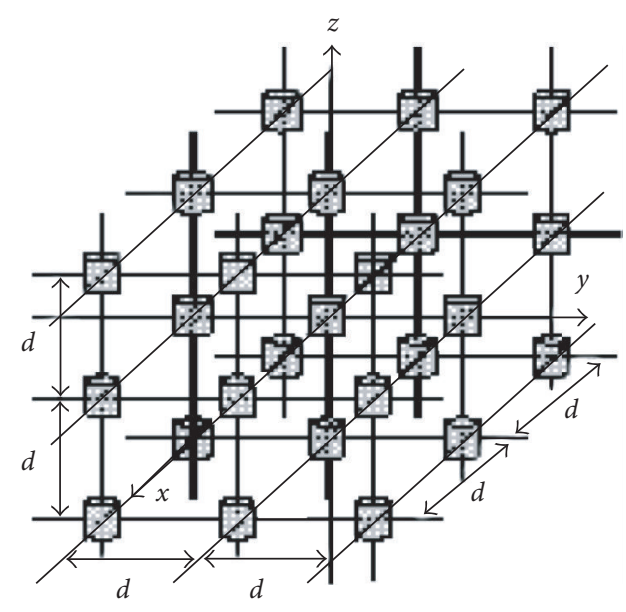

(a) Uniform distribution of magnetic nanoparticles

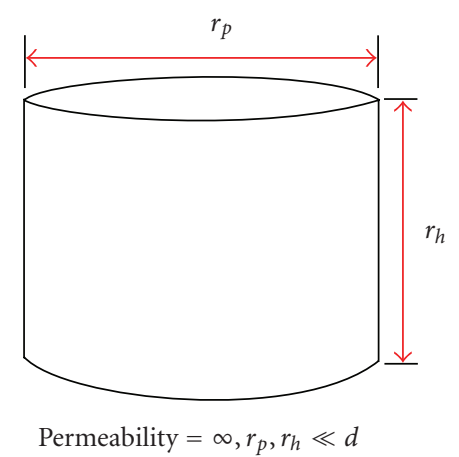

(b) Close-up view of a single magnetic nanoparticle

FIGURE 4: Model showing magnetic nanoparticles distributed uniformly in magnetic fluid.

The essential parameters of magnetic fluid are

(i) $w f$ : weight rate of magnetic bead in grams per cubic centimeter $(\mathrm{g} / \mathrm{cc})$,

(ii) $D_{w}$ : weight density of the magnetic fluid in milligrams of ferrite per cubic centimeter $(\mathrm{mgFe} / \mathrm{cc})$,

(iii) $D_{v}$ : magnetic fluid volume density measured as a percentage.

Given that $D_{v}$ and $D_{w}$ are small, then the relationship between them can be simplified to

$$
D_{v}=\frac{D_{w}}{\gamma_{f}}
$$

where $\gamma_{f}=4.58$ (W-35 sample, Taiho Co., Japan) and is the specific gravity of magnetic bead.

Table 1 provides the volume and weight densities for different combination of nanoparticles and water. From Table 1, it can be seen that the $D_{v}$ percentage change between the lower weight densities is low. For example, the $D_{v}$ percentage change between $D_{w}$ of 0.00479 and 0.00574 is $0.021 \%$.
TABLE 1: Magnetic fluid parameters.

\begin{tabular}{lccc}
\hline Ratio & $w f(\mathrm{~g} / \mathrm{cc})$ & $D_{w}(\mathrm{mgFe} / \mathrm{cc})$ & $D_{v}(\%)$ \\
\hline $1: 120$ & 0.00481 & 0.00479 & 0.105 \\
$1: 100$ & 0.00576 & 0.00574 & 0.126 \\
$1: 70$ & 0.00820 & 0.00814 & 0.179 \\
$1: 50$ & 0.01141 & 0.01131 & 0.249 \\
$1: 40$ & 0.01420 & 0.01404 & 0.31 \\
$1: 27$ & 0.02079 & 0.02045 & 0.454 \\
$1: 22$ & 0.02530 & 0.02481 & 0.552 \\
$1: 18$ & 0.03063 & 0.02992 & 0.669 \\
$1: 13$ & 0.04157 & 0.04026 & 0.908 \\
$1: 10$ & 0.05291 & 0.05081 & 1.155 \\
$1: 8$ & 0.06467 & 0.06156 & 1.412 \\
$1: 7$ & 0.07275 & 0.06884 & 1.588 \\
$1: 6$ & 0.08314 & 0.07807 & 1.815 \\
$1: 5$ & 0.09700 & 0.09016 & 2.118 \\
$1: 4$ & 0.11640 & 0.10669 & 2.541 \\
\hline
\end{tabular}

So it is essential that the percentage change in uniform magnetic field region be less than this value. An error percentage of $\leq 0.01 \%$ was decided for $0.05 \mathrm{~m}$ in the positive and negative axial direction ( $z$-axis) between the coils, and the same was in the radial direction.

\section{HELMHOLTZ COIL AND EXPERIMENTAL SETUP}

Helmholtz coils are used in a variety of applications, primarily due to its ability to produce a relatively uniform field configuration, ease of construction and flexibility. The accuracy of the relative magnetic field produced depends on how precise the Helmholtz coils are constructed and how accurate the current through them is maintained [4-9]. A helmholtz coil system consists of two coils, either circular or square, of equal radius and equal number of turns along an axis through the centre of the coils, separated by a distance equal to the radius of the coils. The total field produced is the sum of the two coils. The helmholtz coil has many applications in a variety of areas such as bioelectromagnetic/biomedical, diagnostic studies on electron beams, calibration of magnetic instruments and probes and in the study of the magnetic properties of materials.

Figure 5 shows the setup used for measurement of volume density of magnetic fluid; the whole system is inside a 2square Helmholtz coil. The theoretical distribution of magnetic flux is shown in Figure 6.

The Helmholtz coil system is used to produce a magnetic flux density of $\mathrm{B}_{0}$. The flux density will concentrate on the magnetic fluid-filled cavity. The needle-type sensor is placed at the center of the cavity, hence the center of the common axis of the coil pairs. The magnetic flux in the cavity can be assumed as $B_{1}$. $B_{1}$ changes with the content density of the magnetic fluid. The theoretical equation estimating the magnetic fluid volume and thus the weight density from the 


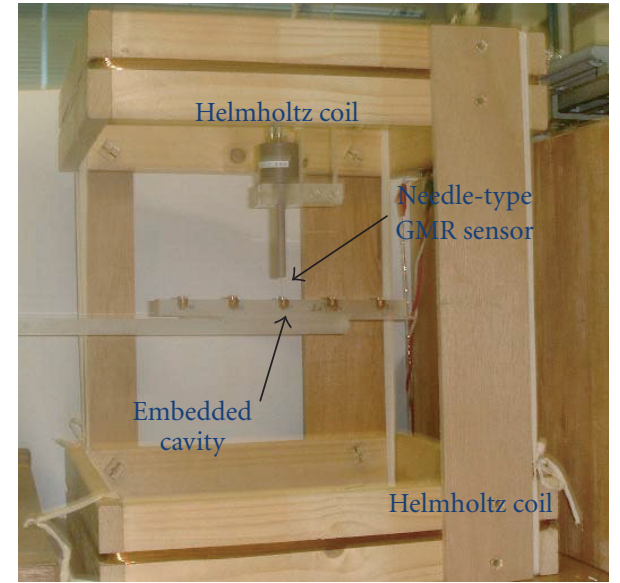

FIgURe 5: A 2-square Helmholtz coil system.

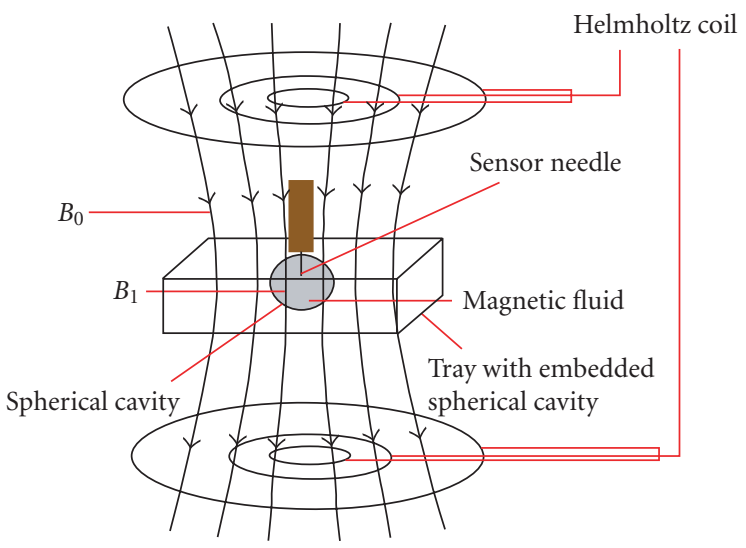

Figure 6: Model of magnetic flux distribution produced by Helmholtz coils and the flux distribution inside an embedded cavity.

difference between the applied flux and the flux in the cavity is [10]

$$
\frac{B_{1}-B_{0}}{B_{0}} \cong 4(1-N) D_{v}
$$

The change between the applied magnetic flux and the flux in the container is directly proportional to the magnetic fluid volume density. The change is usually of the order of $10 \%-$ $12 \%$.

The above equation is based on the assumption that the 2-square Helmholtz coils produce a uniform magnetic field distribution. But in practice, the magnetic field distribution is not very uniform as shown in Figure 7. So a new coil system has been explored and developed.

\section{DEVELOPMENT OF A NEW HELMHOLTZ COIL SYSTEM}

A Helmholtz coil system has been designed and constructed to obtain a uniform magnetic field distribution with an estimated error $\leq 0.01 \%$ for $0.05 \mathrm{~m}$ in the $z$ and $r$ axes. After a lot of discussions to meet the specifications and experimental setup, the ideal system was decided as a planar coil system consisting of three coil pairs. The intended coil is shown in Figure 8 . The three-coil system is used to produce a uniform magnetic flux distribution inside the region of interest.

The magnetic flux distribution as well as the comparison (error in Figures 9 and 10) of the magnetic flux density at any point with respect to the center has been calculated analytically. Figures 9 and 10 show the surface plot and contour plot of analytical results for Helmholtz tri-coil system. It is seen that the error margin with the region of interest is negligible.

The magnetic flux density distribution inside Helmholtz tri-coil system has been measured using a gaussmeter (MTIHM315). An area of $400 \mathrm{~mm}^{2}$ in the uniform region was tested for a $Z$-direction range from $-20 \mathrm{~mm}$ to $+20 \mathrm{~mm}$. However it must be noted that the display of the gaussmeter was accurate only to $0.01 \%$ and the error in the system was approximately $1 \%$. Figures 11 to 13 show measured error margin of contour plot at different positions.

\section{EXPERIMENTS AND EXPERIMENTAL RESULTS}

Helmholtz tri-coil system has been used for providing magnetic flux. Magnetic fluid is injected into a tray with embedded cylindrical cells as shown in Figure 5. The SV-GMR needle sensor tip is set-up in the center of the Helmholtz coil. The tray is moved to get the needle in the middle of the magnetic fluid-filled cylindrical shell. The function generator provides the AC excitation current with the Helmholtz coil to produce a magnetic flux density of $100 \mu \mathrm{T}$. The frequency of operation is set at $100 \mathrm{~Hz}$ and $200 \mathrm{~Hz}$, respectively. Constant current of $0.5 \mathrm{~mA}$ is applied to the SV-GMR sensor. The output of the GMR sensor is obtained by the NF Electronic Instruments 5610B two-phase lock in the amplifier and shown in Figure 14.

The experiments above were performed with the tip of the needle sensor placed at the middle of the magnetic fluid. Experiments were then performed to see the effect of penetration depth ( $y$-direction) on the output. The model is shown in Figure 15.

Figure 15 above shows the needle sensor at the start $(0 \mathrm{~mm})$. This is when the needle sensor initially comes into contact with the magnetic fluid. The fluid is filled with up to $1 \mathrm{~cm}$ of the cylinder. The center is then $5 \mathrm{~mm}$ from the start. Between the start and the middle, four points are tested in $1 \mathrm{~mm}$ steps, that is, at $1 \mathrm{~mm}, 2 \mathrm{~mm}$, and until $5 \mathrm{~mm}$. After the middle, a further two steps are tested. Four concentrations from the 15 samples are tested (two extremes and two from the middle). Figures 16 and 17 show the variation of the change of magnetic flux density as a function of penetration depth of the needle sensor for $100 \mathrm{~Hz}$ and $200 \mathrm{~Hz}$, respectively. It is seen that the signal increases with the increase in penetration depth in the beginning and attains a maximum value and then it decreases slightly and reaches a steady state value. The value for $200 \mathrm{~Hz}$ is slightly less than $100 \mathrm{~Hz}$. The reason of the increase of change linearly in the beginning is that first the sensor creates an indentation while it touches the magnetic liquid and then the sensor gets more magnetic 


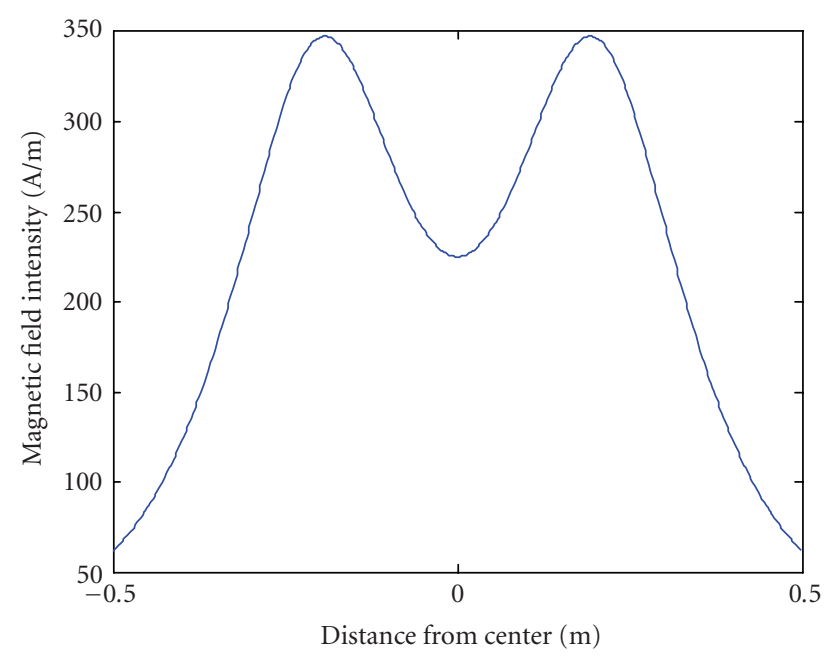

(a) Analytical calculation

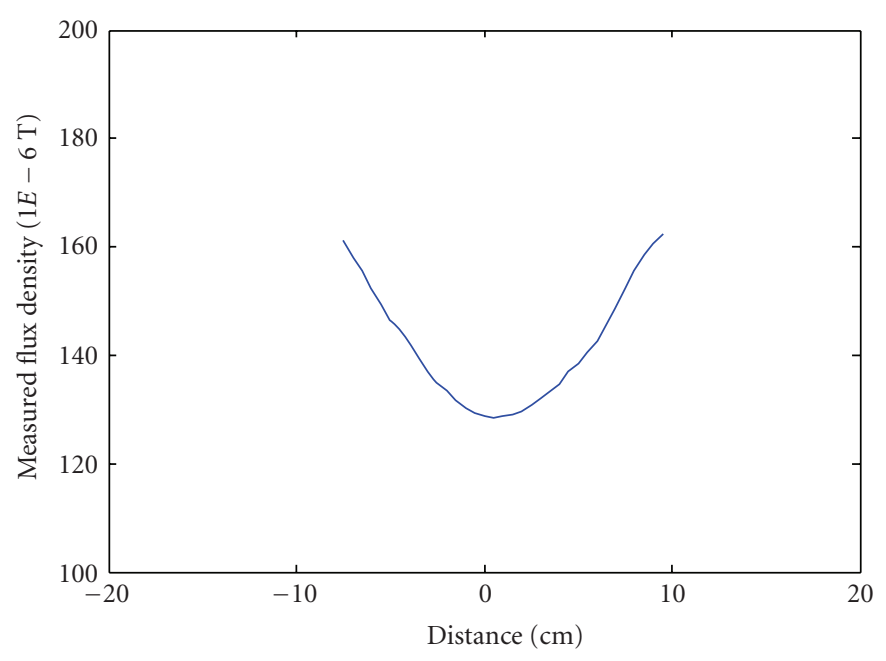

(b) Measured value

Figure 7: Magnetic field distribution inside a 2-square Helmholtz coil system.

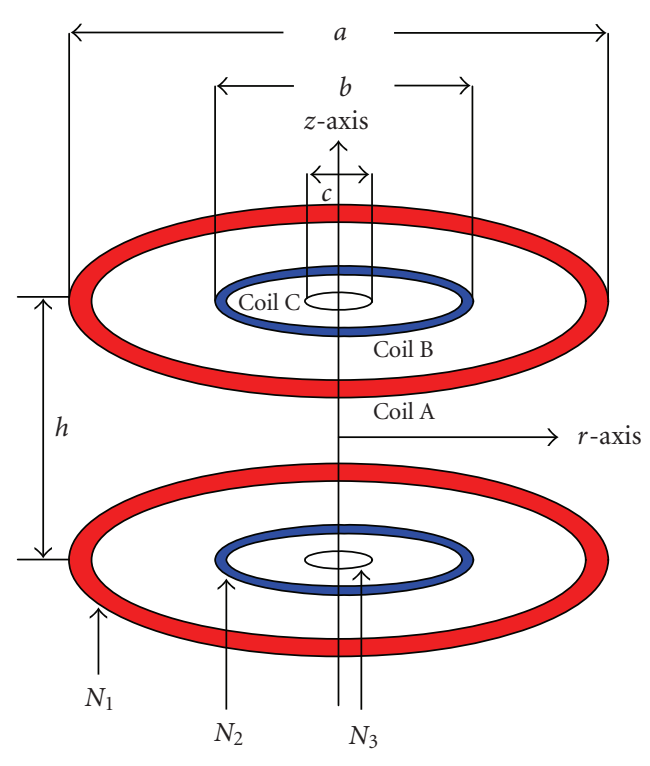

(a) Diagram

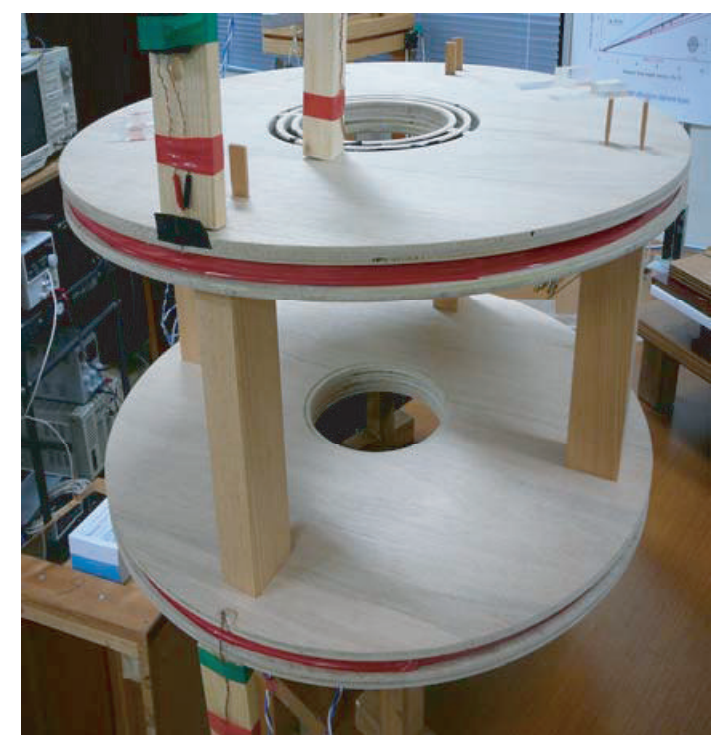

(b) Fabricated system

FIgURE 8: Three-coil pair system Helmholtz coil.

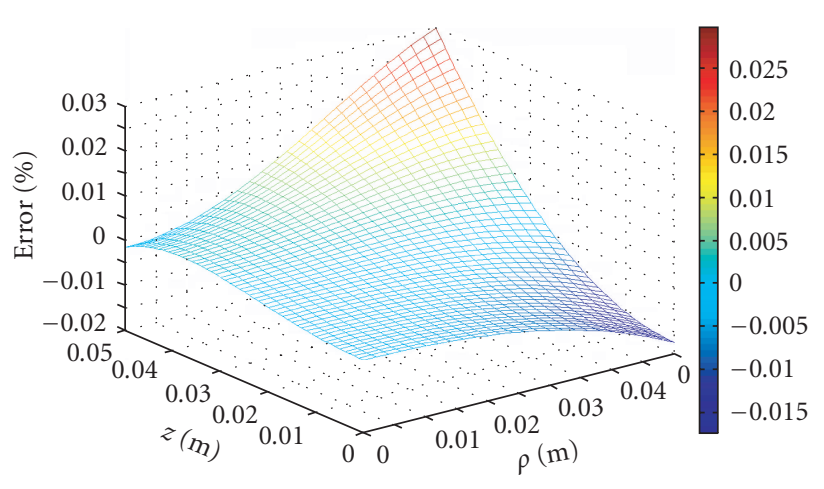

FIGURE 9: 3D surface plot of error in the $z$ and $\rho$ axes.

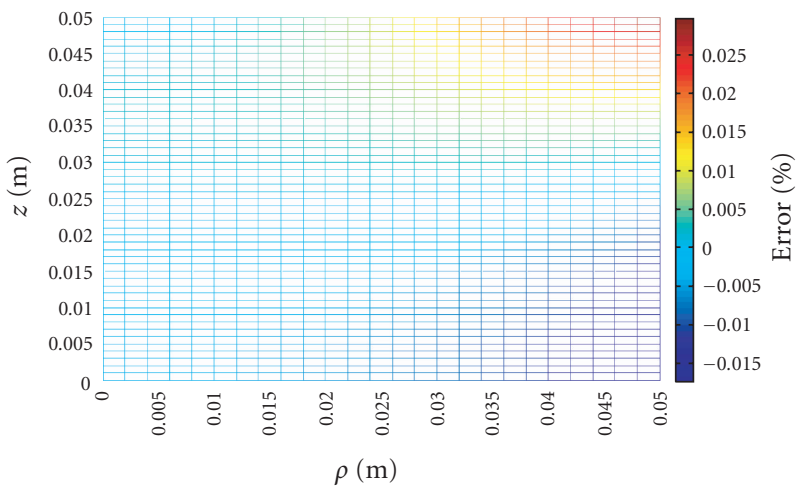

FIgURE 10: Contour plot of error in the $\rho$ and $z$ axes. 


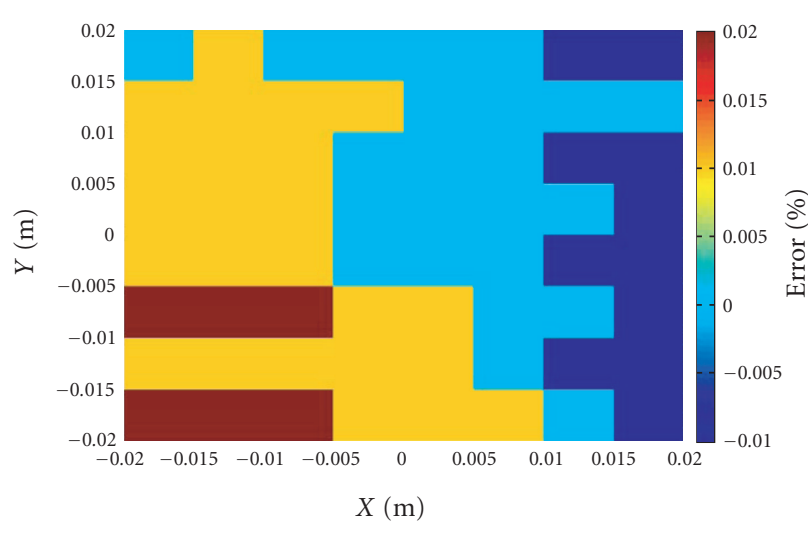

Figure 11: Contour plot at $Z=0 \mathrm{~mm}$.

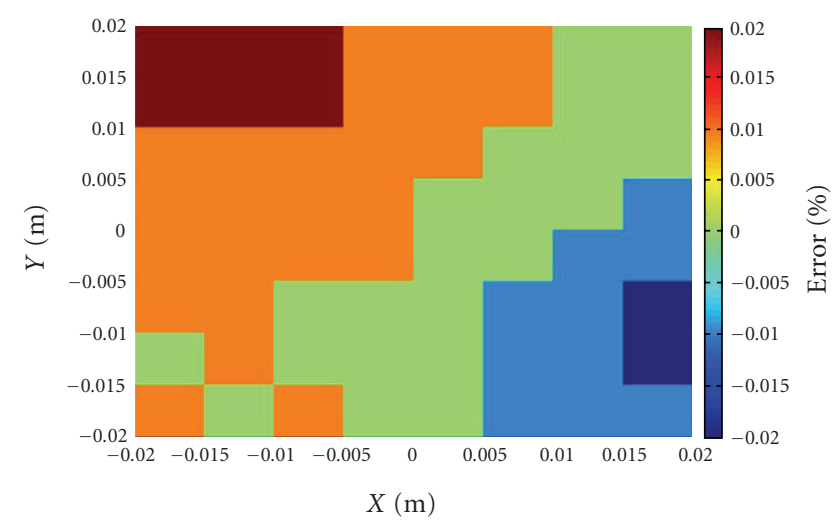

Figure 12: Contour plot at $Z=+20 \mathrm{~mm}$.

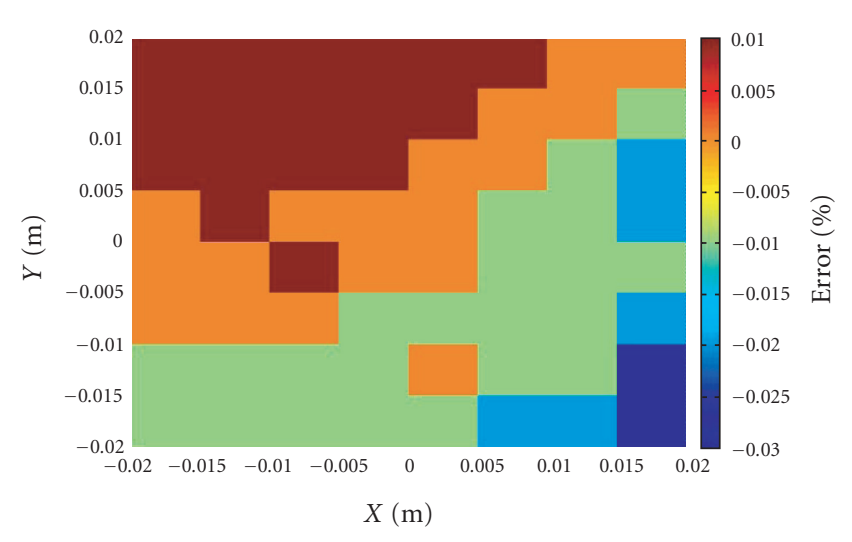

Figure 13: Contour plot at $Z=-20 \mathrm{~mm}$.

material with the increase in the depth of the needle. When the sensor is just inside the magnetic fluid, there is an effect of crowd of magnetic flux at the upper layer of the magnetic fluid. With the increase in depth, the edge effect is negligible.

In this study, the volume (or weight) density of the magnetic fluid has been estimated by the needle-type GMR sensor. In practical situation, it is essential to know the actual temperature. In order to obtain the temperature accurately,

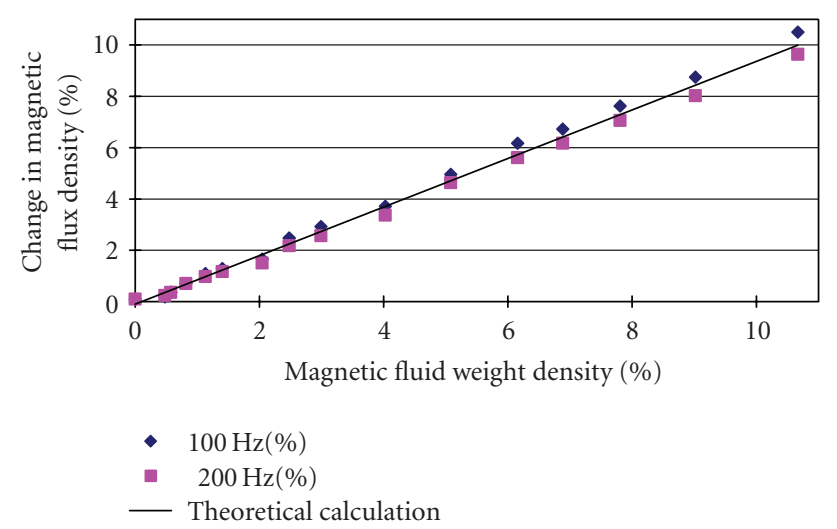

FIGURE 14: Change in magnetic flux density for varying fluid weight density.

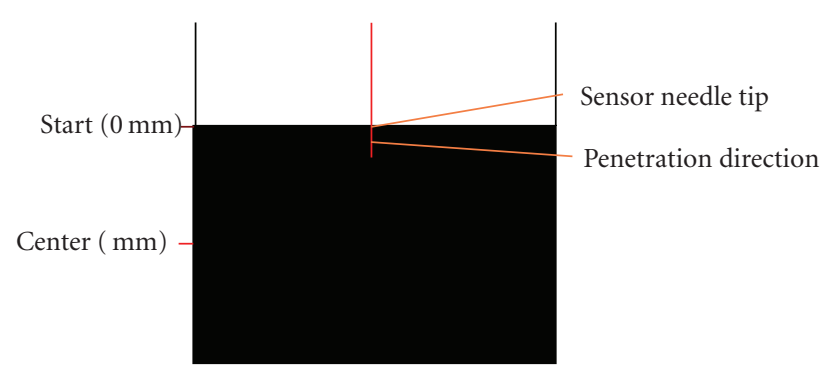

Figure 15: The model showing the effect of penetration depth.

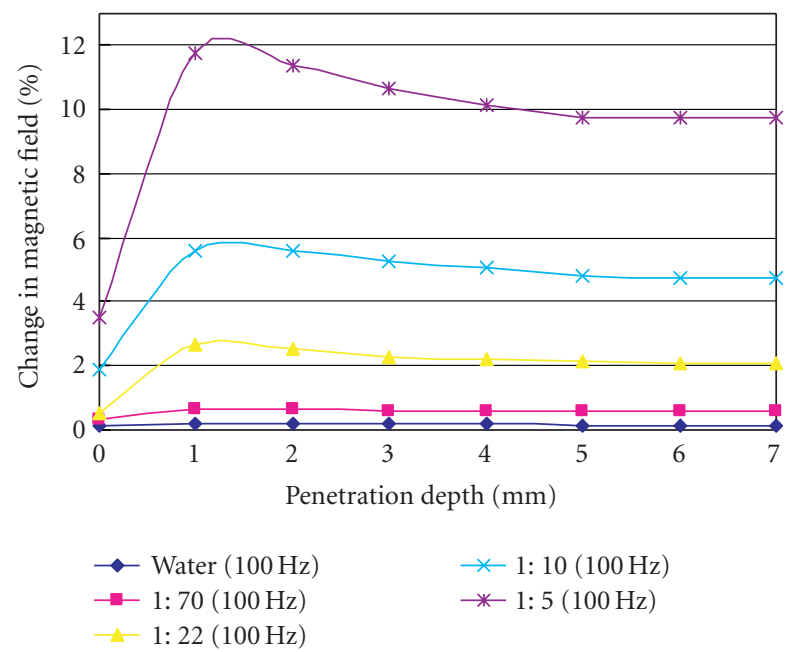

Figure 16: Magnetic field change for penetration depth at $100 \mathrm{~Hz}$.

it is very important to know the volume density of the magnetic fluid. So by using the needle-type GMR sensor, the volume density has been estimated on trial. While researches into the heating effect on cells have been carried out for a long time, it is only recently that safe methods have been found to increase the temperature in treatment or in vivo trials. Numerous trials have been carried out on the relationship between temperature and dextran magnetite and 


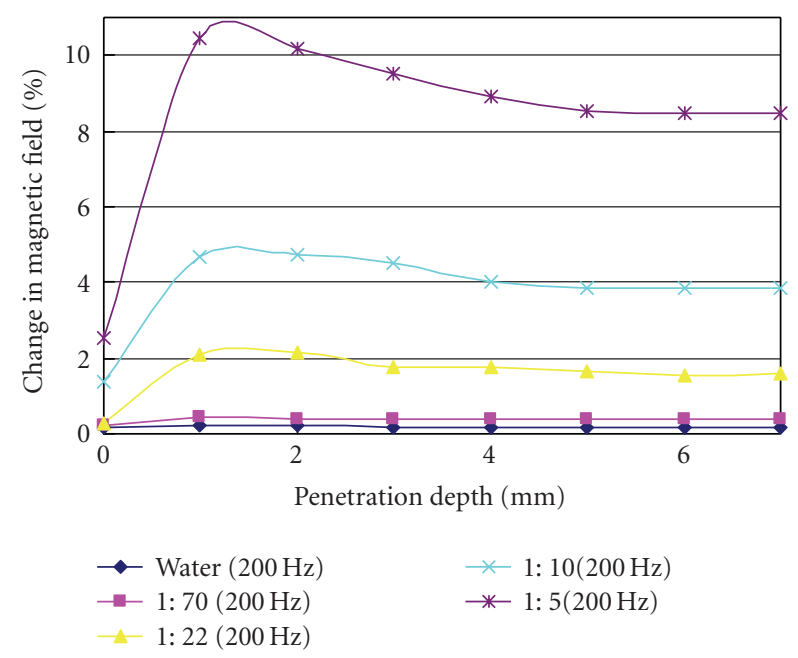

Figure 17: Magnetic field change for penetration depth at $200 \mathrm{~Hz}$.

reported in literature with very encouraging results [14-18]. Furthermore, there is a widespread belief that hyperthermia has the potential to be an effective treatment, validated by the fact of the number of clinics that carry out such treatment currently. However the side effect of possible damage to healthy cells means that an effective and accurate method is needed to estimate the magnetic fluid volume density. The SV-GMR sensor can be utilized in such a way to estimate the magnetic fluid volume density in vivo paving the way for less/no side effects for patients, as well as the widespread possibility of being a stand-alone cancer therapy rather than an adjunct to other cancer treatments, which seems to be the popular practice at the moment.

\section{CONCLUSION}

A needle-type spin-valve giant magneto-resistive(SV-GMR) sensor has been developed for measurement of flux density inside human body. It is important to estimate the volume density of magnetite inside the body for the hyperthermia cancer treatment based on induction heating. A new Helmholtz three-pair coil system has been developed for providing uniform flux distribution in the region of interest. The relationship between the difference of magnetic flux density and magnetic fluid volume density contained in an embedded cavity has been obtained and it is linear. It can therefore be concluded that the volume density of magnetite injected into the body may be estimated by the proposed technique.

\section{REFERENCES}

[1] G. L. Cote, R. M. Lec, and M. V. Pishko, "Emerging biomedical sensing technologies and their applications," IEEE Sensors Journal, vol. 3, no. 3, pp. 251-266, 2003.

[2] R. A. Yotter, L. A. Lee, and D. M. Wilson, "Sensor technologies for monitoring metabolic activity in single cells - part I: optical methods," IEEE Sensors Journal, vol. 4, no. 4, pp. 395-411, 2004.
[3] R. A. Yotter and D. M. Wilson, "Sensor technologies for monitoring metabolic activity in single cells_-part II: nonoptical methods and applications," IEEE Sensors Journal, vol. 4, no. 4, pp. 412-429, 2004.

[4] C. C. Berry and A. S. G. Curtis, "Functionalisation of magnetic nanoparticles for applications in biomedicine," Journal of Physics D, vol. 36, no. 13, pp. R198-R206, 2003.

[5] J. L. Kirschvink, "Uniform magnetic fields and doublewrapped coil systems: improved techniques for the design of bioelectromagnetic experiments," Bioelectromagnetics, vol. 13, no. 5, pp. 401-411, 1992.

[6] K. Kakuno and Y. Gondo, "Three and four square coil systems for producing uniform magnetic fields," Bulletin of the Faculty of Engineering, Yokohama National University, vol. 25, pp. 179192, 1976.

[7] R. S. Caprari, "Optimal current loop systems for producing uniform magnetic fields," Measurement Science and Technology, vol. 6, no. 5, pp. 593-597, 1995.

[8] J. Wang, S. She, and S. Zhang, "An improved Helmholtz coil and analysis of its magnetic field homogeneity," Review of Scientific Instruments, vol. 73, no. 5, pp. 2175-2179, 2002.

[9] G. Gottardi, P. Mesirca, C. Agostini, D. Remondin, and F. Bersani, "A four coil exposure system (tetracoil) producing a highly uniform magnetic field," Bioelectromagnetics, vol. 24, no. 2, pp. 125-133, 2003.

[10] S. C. Mukhopadhyay, K. Chomsuwan, C. P. Gooneratne, and S. Yamada, "A novel needle-type SV-GMR sensor for biomedical applications," IEEE Sensors Journal, vol. 7, no. 3, pp. 401-408, 2007.

[11] S. C. Mukhopadhyay, "A novel planar mesh-type microelectromagnetic sensor-part I: model formulation," IEEE Sensors Journal, vol. 4, no. 3, pp. 301-307, 2004.

[12] S. C. Mukhopadhyay, "A novel planar mesh-type microelectromagnetic sensor-part II: estimation of system properties," IEEE Sensors Journal, vol. 4, no. 3, pp. 308-312, 2004.

[13] S. C. Mukhopadhyay, C. P. Gooneratne, G. S. Gupta, and S. N. Demidenko, "A low-cost sensing system for quality monitoring of dairy products," IEEE Transactions on Instrumentation and Measurement, vol. 55, no. 4, pp. 1331-1338, 2006.

[14] J. van der Zee, "Heating the patient: a promising approach?" Annals of Oncology, vol. 13, no. 8, pp. 1173-1184, 2002.

[15] B. Hildebrandt, P. Wust, O. Ahlers, et al., "The cellular and molecular basis of hyperthermia," Critical Reviews in Oncology/Hematology, vol. 43, no. 1, pp. 33-56, 2002.

[16] P. Wust, B. Hildebrandt, G. Sreenivasa, et al., "Hyperthermia in combined treatment of cancer," The Lancet Oncology, vol. 3, no. 8, pp. 487-497, 2002.

[17] R. Hergt, W. Andrae, C. G. d'Ambly, et al., "Physical limits of hyperthermia using magnetite fine particles," IEEE Transactions on Magnetics, vol. 34, no. 5, part 2, pp. 3745-3754, 1998.

[18] Y. Yamazaki, K. Igarashi, M. Tomita, et al., "Heating characteristics of dextran magnetite under a strong AC magnetic field created by a portable magnetic generator," in Proceedings of the Asia-Pacific Radio Science Conference (AP-RASC '01), p. 412, Chuo University, Tokyo, Japan, August 2001. 

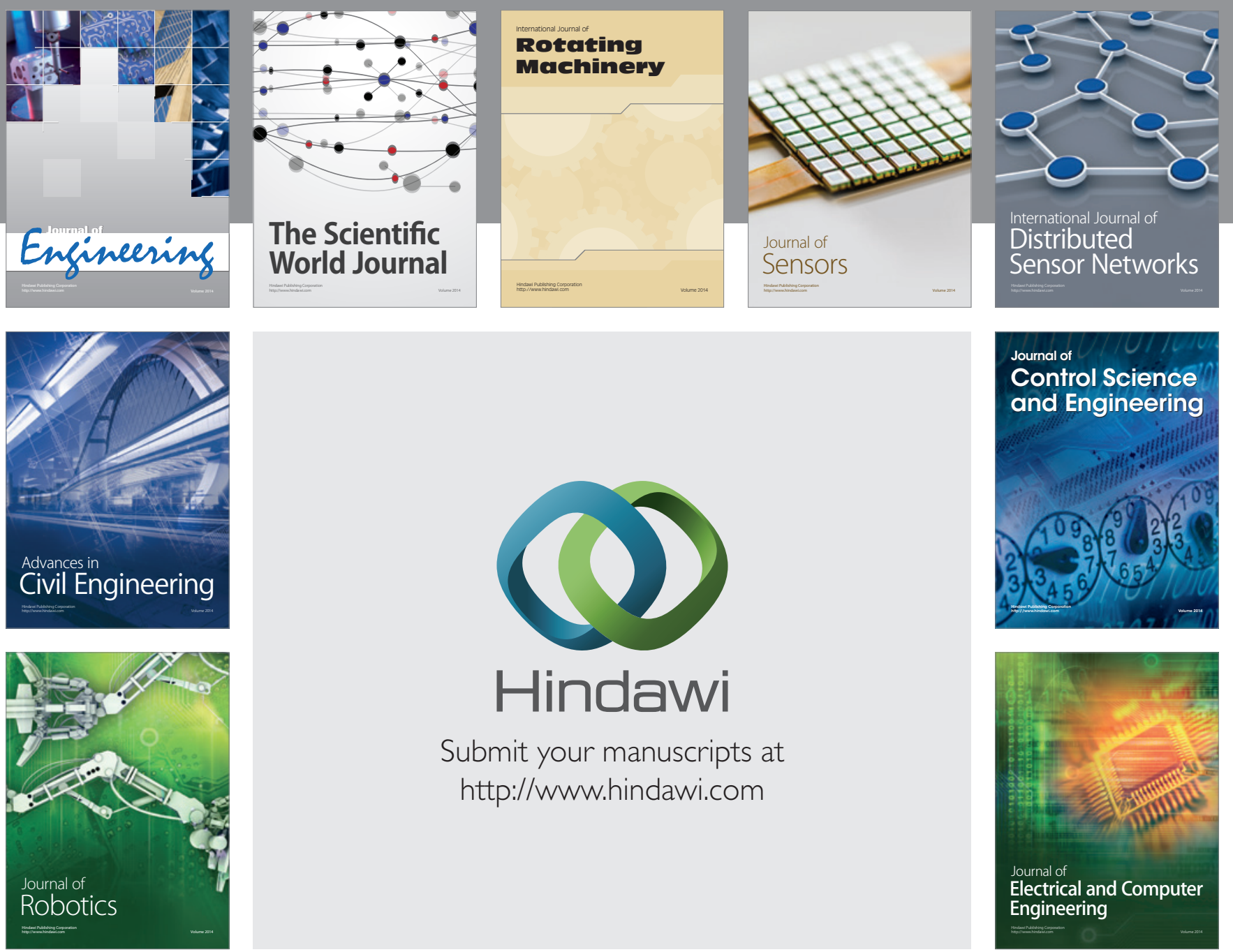

Submit your manuscripts at

http://www.hindawi.com
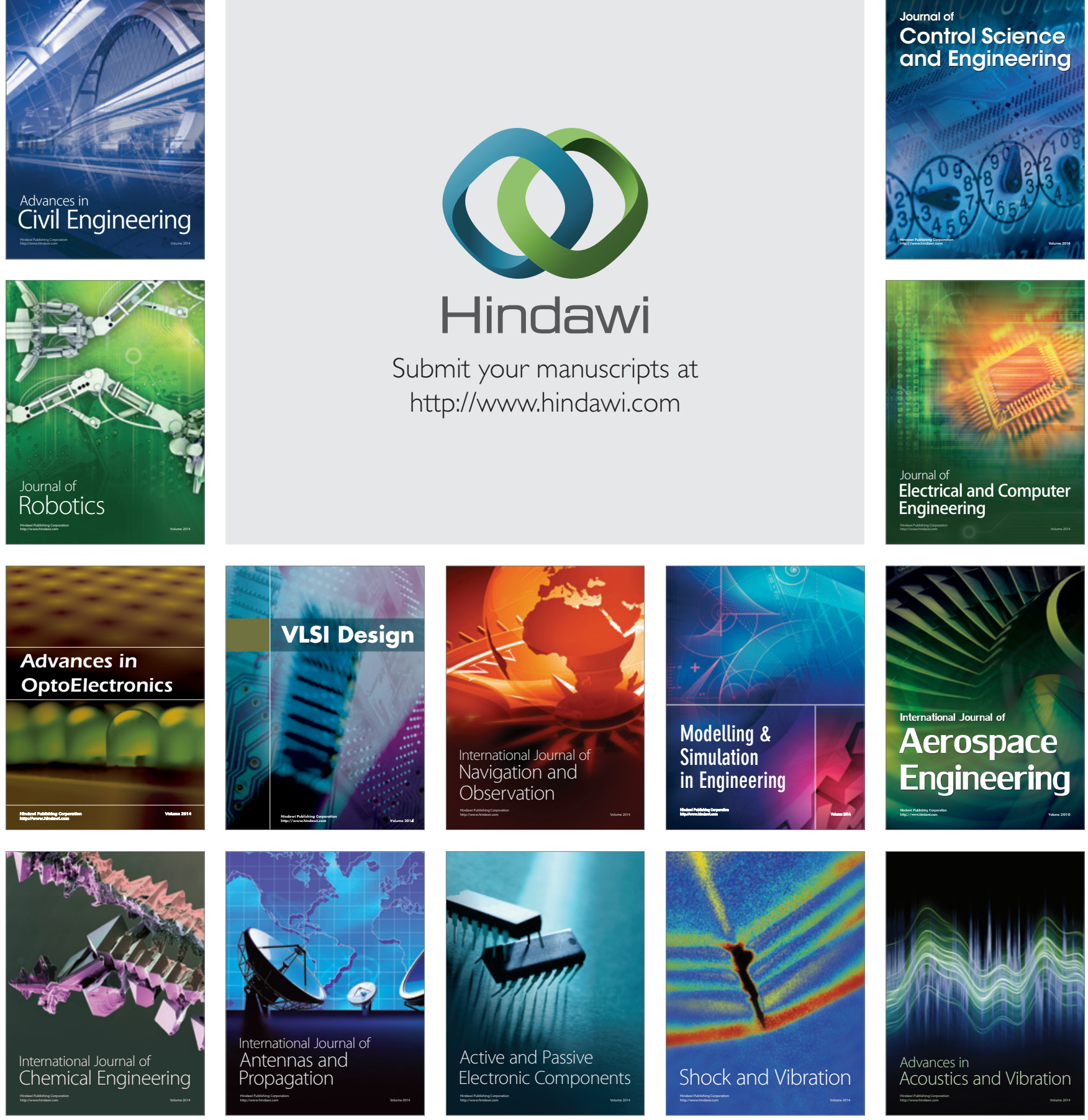